\title{
Dynamics, Stability, and Control of Stepping
}

\author{
Hooshang Hemami, ${ }^{1}$ Kamran Barin, ${ }^{2}$ LaCi Jalics, ${ }^{3}$ and Deborah Givens Heiss ${ }^{4}$ \\ ${ }^{1}$ Department of Electrical and Computer Engineering, The Ohio State University, Columbus, OH 43210; ${ }^{2}$ Department of \\ Otolaryngology, The Ohio State University, Columbus, OH 43210; ${ }^{3}$ Delphi Automotive, 51786 Shelby Parkway, Shelby, MI 48315; and \\ ${ }^{4}$ Physical Therapy Division, The Ohio State University, Columbus, OH 43210
}

(Received 23 October 2003; accepted 14 April 2004)

\begin{abstract}
The dynamics, stability, and control of stepping are considered. The role of internal models is elaborated.

The main objective of the paper is to provide a better understanding of the machinery and processing in the central nervous system (CNS) that relates to stepping. The role of the vestibular system in balance and balance recovery is described. Balance and balance recovery are essential in stepping, and guarantee the stability of the system before, during, and after stepping. In sagittal standing, humans use two distinct sets of control strategies to maintain their postural stability in response to external disturbance. In one set of strategies, the configuration of the base of support, namely, the position of the feet, remains unchanged. The ankle and hip strategies are examples of postural adjustments where the feet do not move. When the disturbances are large, and move the center of mass or pressure outside the support boundaries, stepping strategies are required. A simple control strategy is proposed for illustrative purposes. Its effectiveness is verified by computer simulation of a seven-link two-dimensional sagittal biped. The applications of the model in assessing trauma and injury are discussed.
\end{abstract}

Keywords-Stepping, Internal dynamics, Vestibular processing postural stability, Stepping, Balance, Control, Motor control.

\section{INTRODUCTION}

It is hypothesized that humans use a continuum of strategies to maintain balance. These strategies range from those in which the feet remain on the ground to those in which one foot is moved to increase the size of the support base. ${ }^{13,26,32}$ Different terminologies describe these two types of strategies: corrective versus protective, fixedsupport versus change-in-support, feet-in-place versus stepping, etc. The feet-in-place strategies have been studied by Nashner and $\mathrm{McCullum}^{26}$ among others. Kuo and Zajac ${ }^{22}$ have developed an analytical model to study coordination of feet-in-place strategies. Using the set of feasible accelerations for different stances, they were able to assess the efficiency of various control strategies. According to their

Address correspondence to Hooshang Hemami, Department of Electrical and Computer Engineering, The Ohio State University, Columbus, OH 43210. Electronic mail: hemami@ece.eng.ohio-state.edu findings, the hip strategy was most effective in moving the center of mass with minimal muscle input.

If the disturbances are larger and tend to move the center of mass or pressure outside the support boundaries, stepping strategies may be utilized that change the support envelope by widening the support boundaries, namely, by taking appropriate steps..$^{25,35}$ Stepping, as protection from falling, has been studied for people of different ages in Refs. 24 and 31. The control machinery involved in stepping, i.e., unloading the appropriate foot such that there is no weight on it, moving the foot quickly to the appropriate location, and reloading it have been discussed in Refs. 11 and 19. In all the above strategies, the movement of the center of mass and the center of pressure ${ }^{18,29}$ must be computed or estimated.

Maki et al., ${ }^{25}$ Thelen et al., ${ }^{35}$ and Rogers et al. ${ }^{30}$ have examined the effect of aging on the characteristics of the stepping strategy to prevent falls. Maki et al. provide a comprehensive review of the studies of the configurations in which the support surface changes. The main conclusion is that the characteristics of the compensatory stepping, in response to disturbances, are quite different from those encountered during volitional movements such as initiation of gait. Furthermore, the use of the stepping strategy is much more common in our daily activities, and the change in the support configuration occurs well in advance of the center of pressure reaching the boundaries of stability.

Presently, our understanding of the control mechanisms involved in compensatory stepping is limited. From a biomechanical viewpoint, stepping entails a complex process requiring the unloading of the appropriate foot such that there is no weight on it, moving it quickly to a new location, and reloading it to preserve postural stability. ${ }^{16}$ Some investigations lead to the assessment that the stepping responses are regulated by preprogrammed motor patterns operating as an open-loop system. However, Maki and McIlory cite the ability of subjects to terminate the stepping response just prior to lifting the foot, as a strong evidence that sensory feedback is necessary and used to modify the response characteristics. Regardless of the exact control mechanisms used, the movements of the center of mass and center of 
pressure appear to be important parameters in determining the timing, the direction in the medial plane, and the size of stepping responses.

For the purposes of voluntary or compensatory stepping under disturbance, as is shown later in the paper, contact conditions of the feet with the ground, velocities of the feet, and the (vector) velocity and the position of the center of mass are needed. One could refer to these needed variables as a subset of the states of the system. ${ }^{4}$

The neural machinery of the CNS, or the internal models, have four major functions in stepping:

1. direct or indirect computation or estimation of the above subset of states;

2. detection of the conditions necessary for taking a corrective step;

3. modulation of the stepping machinery; size, direction, and initiation; and finally

4. implementation of the stepping.

All of the above items may involve fusion of the sensory modalities of proprioception, vestibular, and vision, ${ }^{15}$ and may require internal models. However, such fusions are not considered in this paper. There is another point that merits mentioning here. Stereotyped signals that the brain uses are considered to be symbols that do not resemble in any way the external world they represent (chapter 1 in Ref. 27). An essential task is to decode the significance of such signals. Psychophysical experiments ${ }^{7}$ point to cases where the internal processing seems to be governed by laws of physics as observed and recorded internally by the human observer. We plan to model the internal processing mechanisms with the physical external models that are known to biomechanics.

\section{CONSIDERATION AND DESCRIPTION OF THE MODEL}

Few models have been developed to study both feet-inplace, and stepping strategies, or the transition from one to the other. In stepping, as envisioned in this paper, one must accommodate rapid changes in the system constraints as the feet progress from full contact to standing on tiptoes, to a one-leg support and the thrust of the other foot forward, and finally to the reestablishment of contact for a new support base.

We provide one example of a controller based on the velocities of both the feet and the center of mass. First, we will discuss how the CNS may perform the necessary measurements, computations, and implement decisions. Second, we will develop a simple control strategy and study its feasibility via computer simulations of a seven-link sagittal biped model.

\section{Internal Model}

Most of the internal models applied to natural systems fall into two categories: internal-variable-based, or externalvariable-based. The primary difference between the two is the choice of physical state variables, and the implication of the laws of nature. With external variable models, one applies laws of nature that are somewhat established. With internal variable models, one does not know all the laws and rules. Further, with all the feedback, feedforward, delays, and dense packing, identification of and access to specific inputs and outputs of an internal model become problematic. As an example, let us consider the head, and consider its attitude, i.e., the rotational motion of the head. The two types of internal models are as follows: In a simple internal model, the angular velocities, as measured in the labyrinth system, and the time-integral of these angular velocities could constitute the six velocity and position variables. The otoliths also provide gravity-based position information such as the angle of the head relative to an inertial frame. The second internal model could be based on the Euler or Bryant angles, relative to an inertial frame, as positione states and the rate of change of these angles with respect to time as velocity states.

Now consider the translational motion of the whole body or the head. Three Cartesian positions and three translational velocities are needed to specify the motion of one point of the body in space. For external variables models, these six variables can be somewhat arbitrary: the motion of the center of mass, center of mass of the torso, etc. An alternative internal variable set of state variables are the time integral of the otolith outputs as velocity variables and the time integral of these velocities as position variables.

Similar integration with respect to time allows one to construct translational and rotational velocity and position of the head and torso from vestibular organ outputs. Ref. 2 (p. 53) discusses leaky integrators. The same kind of argument could be applied to construction of neural circuits that have very slow leakage, or, alternatively stated, very long time constants, and thus become approximate integrators (with respect to time). Is ia also known in system theory that a stable low-pass RC system can be made into an integrator with sufficient positive feedback. The point is, whatever mechanism is used to represent the position variables, it must be able to hold the value for a long time, because humans and animals can stay in static positions for long periods. This necessity for a neural circuit to maintain a variable for a long time may dictate design of internal integrators by pools of neurons that are capable of such behavior. Horak and Shupert, ${ }^{14}$ on the other hand, have proposed that the vestibular system appears not to contribute to static postures. One can therefore argue that perhaps position variables needed for the stability of static postures are constructed from the proprioceptive system, and are not direct outputs of integrators. It has been demonstrated in 
Ref. 21 that position states can be estimated from neural networks whose only inputs are the spindle (position and velocity) signals. Of course, since different spindle signals arrive at the CNS computation centers with different latencies, predictive-corrective ${ }^{34}$ neural circuits are called for to compensate for the delays. Setting the delay issue aside for the time being, the fact is that, in general, the vector velocities of any point of the seven-link biped model can be derived from the knowledge of the state and the geometry of the position of the point. ${ }^{10,21,28}$ The states, as said before are, in turn, estimated from the totality of spindle signals.

\section{Stability}

It is well known that the stability of musculoskeletal systems can be brought about by coactivation that amounts to limb position and velocity feedback. It is important to present a possible second application of vestibular signals in torso stability and control. The role of the vestibular system in head position control, ${ }^{30}$ and vestibulo-ocular reflex ${ }^{1}$ is well known. When the neck muscles are voluntarily made very stiff, the head, the neck, and the body act as one single rigid body. In this situation, it is very easy for the CNS to control the torso by the same head control strategy. Another situation is when the head is restrained relative to the torso. ${ }^{14}$

The above discussion is for the purpose of simply illustrating how the vestibular machinery may be utilized in estimating the center of mass. In reality, the CNS uses the vestibular machinery and the lengths and velocities of the neck as well as other muscles in estimating the center of mass rather than insisting on stiffening the neck muscles. However, this more realistic estimation method is not implemented here. A partial estimation method where the lengths and velocities of muscles are used to estimate the angular positions and angular velocities of the head and the torso is implemented in Ref. 21.

Cannon et al. ${ }^{6}$ have discussed a neural network integrator in the vestibulo-ocular reflex path of labyrinth signals. It is argued here that the same type of integrator should be involved in the processing of the vestibulospinal paths for torso control. Dietz et al. ${ }^{8}$ discuss how the vestibulospinal reflexes play a major role in regulation of body sway by correcting for small disturbances of the trunk before the stretches of the leg muscles become significant, and start to exert their control.

Kawato ${ }^{20}$ has discussed how the three variables of acceleration, velocity, and position are used in combination ot excite motor neurons of the eye muscles. As a first step, therefore, one would look for similar combinations for controlling the muscles of the neck and the torso.

The otolith organs are involved in the stability and in the establishment of a frame of reference for the body. There is a static component and a dynamic component to the otolith output. The static component is for position sensing. The otolith organs can determine the pitch and roll directly. ${ }^{5,36}$
The yaw angle could be measured by the vision system and the neck receptors. The dynamic component of the otolith output could be measuring the angular velocity for a small bandwidth near zero to complement the frequency range of the semicircular canals. ${ }^{3}$

It is shown (Ref. 36, pp. 826-827) that the vestibular signals in the rabbit and cat extend down to the cervical, thoracic, and lumbar spine, and that there are effects of both the canal and otolith signals. The presence of both signals signifies position and velocity control. Their distal extension signifies that they may be involved in the postural stability of the trunk as well. At that level, they would have effects such that the trunk is brought towards its vertical equilibrium relative to the thighs and hips. The effect of such feedback in the form of vestibular reflexes, i.e., the "vestibulocollic reflex," has been discussed on page 18 in Ref. 9. Furthermore, if all the joints were locked, the effect of this feedback of position and velocity should be felt at the ankle joint for postural stability of the whole body as an inverted pendulum.

\section{Linear Acceleration Sensors, and Triggering Function}

One can stipulate at least four uses of the linear acceleration sensors, namely, gravity compensation, estimation of the torso center of mass, awareness of one's translational position in space, and triggering and controlling stability. We assume that is how the torso center of mass is sensed.

The dynamic component of this sensor is used to estimate the acceleration of the head and, when integrated with respect to time, to provide the linear velocity of the head. The linear velocity and position of the head could be used by the CNS in computation of the velocity and position of the center of mass of the torso. A simple case of this situation is elaborated here. Suppose the neck muscles are very stiff, so that the head and the torso move as one rigid body. Furthermore, let us assume that the person is sitting on a chair, so that the torso and head can be modeled as an inverted pendulum. ${ }^{12}$ Suppose further, that the center of mass of the torso lies on a straight line from the pivot on the chair to the otolith organs. The linear position and velocity of the torso are a fraction of those measured in the head, and the fraction can be learned in a neural network learning scheme. Since the position of the head is available as a result of double integration of the linear acceleration, the position information could give one a sense of one's position in space, for example, as when one closes one's eyes and jumps straight up. Another use of the linear velocity of the head or the torso relative to the hip is in a class of postural adjustments. If, from an equilibrium standing position, the body is disturbed such that the linear velocity of the torso pushes the center of mass out of the base of support, then the linear velocity could trigger and set in motion a postural adaptation strategy to unload the posterior leg relative to 
the direction of motion and throw it in the direction of torso motion to prevent falling. This is stepping that is triggered by requirements of balance and postural stability. The level of the trigger is assumed to be available from learning and experience.

\section{A Control Strategy}

After a push from behind, three basic strategies [13, 35] are used by humans to keep from falling: bending at the knees and hips, bending at the ankles, or stepping forward.

One particular control strategy is explored here. It is a strategy that, under the constraints and conditions described here, works as shown by computer simulations. It could potentially be used by humans to take a step in order to avoid falling. The velocity of the center of mass is controlled. If it is above a certain threshold, one foot is thrown forward to widen the stance. The exact width of the stance depends on the size of the disturbance, and is unimportant as long as a fall is avoided. Once the biped arrives at a stable stance, position feedback is utilized to move the biped's joint angles to a desired location. ${ }^{16}$

The control strategy is to unload the heel (or heels), i.e., getting on tiptoes and, if necessary, take a step forward to prevent falling. The transition from moving up on the toes to the stepping strategy occurs if the center of mass of the biped moves over its toes, initiating a fall or if the speed of the center of mass exceeds a given threshold alarming the person about the impending fall.

Figure 1 shows how the control works. The control has a state of equilibrium, a set of four actions, and five decisions based on computation of certain variables. In the figure,

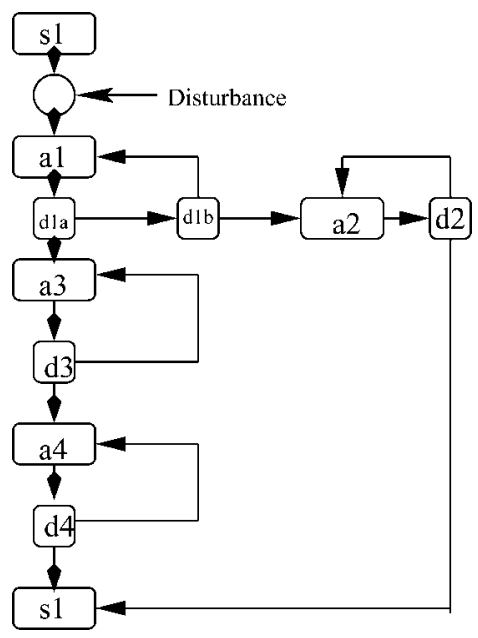

FIGURE 1. The sequence of actions, computations and autonomous decisions is shown. The measurements may be continuous throughout the maneuver. The actions are all precipitated by a state of readiness, i.e., by anticipating the disturbance. the two states, labeled s1, define the position of standing equilibrium. The action states are the following:

a1: The biped lifts up. Toes are loaded, heels are unloaded. The process may absorb kinetic energy, and may lead to in-place foot strategy.

a2: The heels are lowered.

a3: The right foot is unloaded and a step is taken.

a4: The right foot is put down.

The four decision steps are the following:

The first decision has two sequential components:

d1a: position of the center of mass (COM) is computed, and it is decided whether it is beyond the toes.

$\mathrm{d} 1 \mathrm{~b}$ : The velocity of COM is measured, and it is decided whether it is negative.

d2: Forces under the heels are measured in order to decide whether the heels are on the ground.

d3: The velocities of the right foot and COM in the horizontal direction are measured in order to decide whether the threshold is exceeded.

d4: forward velocity of the COM in the horizontal direction is measured in order to decide whether it is negative.

Suppose the biped is standing with its feet together and it receives a disturbance from behind. From a control point of view, several strategies could have been implemented to unload the weight on one foot. For completeness, we briefly describe several of these strategies. They could be implemented singly or as combinations.

1. If a biped moves sufficiently to one side, as a single pendulum in the frontal plane, the foot opposite to the direction of motion gets unloaded.

2. The same motion may be initiated by rotating the foot about the toes, and, consequently, that foot gets unloaded.

3. The foot can be unloaded by hip motion alone.

4. The same motion as in the above step can be brought about by a combination of hip and knee movements.

5. The ankle strategy of unloading the heels first.

We arbitrarily choose the last strategy here. We also assume that the voluntary strategy we develop is the one that is memorized and utilized under unexpected disturbance. The controller first attempts to unload the heels of the biped. There are several ways to doing this. We do one that raises the biped on its toes and lifts its center of mass. This corresponds to using an ankle strategy. For small forward disturbances, rising on the toes will return the biped to its equilibrium. When the disturbance moves the center of mass backwards, rising on toes will not help, but bending the upper torso forward (hip strategy) is more appropriate. Consequently, moving up on the toes may reposition the center of mass to its original state. This corresponds to a combined 
ankle-hip strategy. ${ }^{26}$ The hip strategy may sometimes be used alone for a forward disturbance.

An internal model is used early in the disturbance to estimate the position and velocity of the center of mass. Velocity feedback is then utilized to control the velocity of the center of mass and the velocity of the feet in order to widen the stance. Once the stance is widened, both position and velocity feedback are utilized to reduce the movement of the system to zero about the new equilibrium point. The controller is predominantly a velocity feedback strategy.

\section{Support Phase}

It is assumed that all the disturbances are identifiable, and all desirable postural strategies (i.e., velocities of the heels and the center of mass, under every disturbance) are available. After a disturbance (a push from behind) the biped moves up on its toes, while rotating its body backwards in order to reduce the forward motion of the center of mass. If the push is not large enough, the upward velocity of the heels, $v_{\mathrm{h}}$ is reversed in order to lower the heels back on the ground. The desired velocity of the center of mass is set to zero. Once the heels touch the ground, the desired velocity of the heels are set to zero. The biped has successfully completed a postural strategy. Position feedback may further be needed in order to return the biped to vertical equilibrium stance. ${ }^{17}$

\section{Stepping}

When the velocity of the center of mass is large, stepping is needed to prevent a fall. The right foot is arbitrarily chosen to step forward. The decision to take a step is made early enough to give time to the right foot to step out in front, a predictive element may be necessary here. At the same time the left heel is brought down for full contact. The control during the swing phase of the right foot reduces the velocity of the center of mass by negative feedback, implemented perhaps by coactivation of a system of muscles. Also the right foot must be brought to a desirable attitude for its eventual contact. There is a need for a positional controller to take control of the final position of the biped. ${ }^{28}$

\section{Biomechanical Model}

The strategies are studied here via a seven-link twodimensional sagittal biped model shown in Fig. 2. Problems of stability, control, and terrain and motion adaptations of this model are treated in detail in Ref. 16. The physical parameters of the biped are given in Table 1. More about these parameters can be found in Ref. 23.

\section{RESULTS}

Two simulations are presented here. The first simulation shows the biped recovering from a disturbance by moving on tiptoe and back down. In the second simulation, the dis-

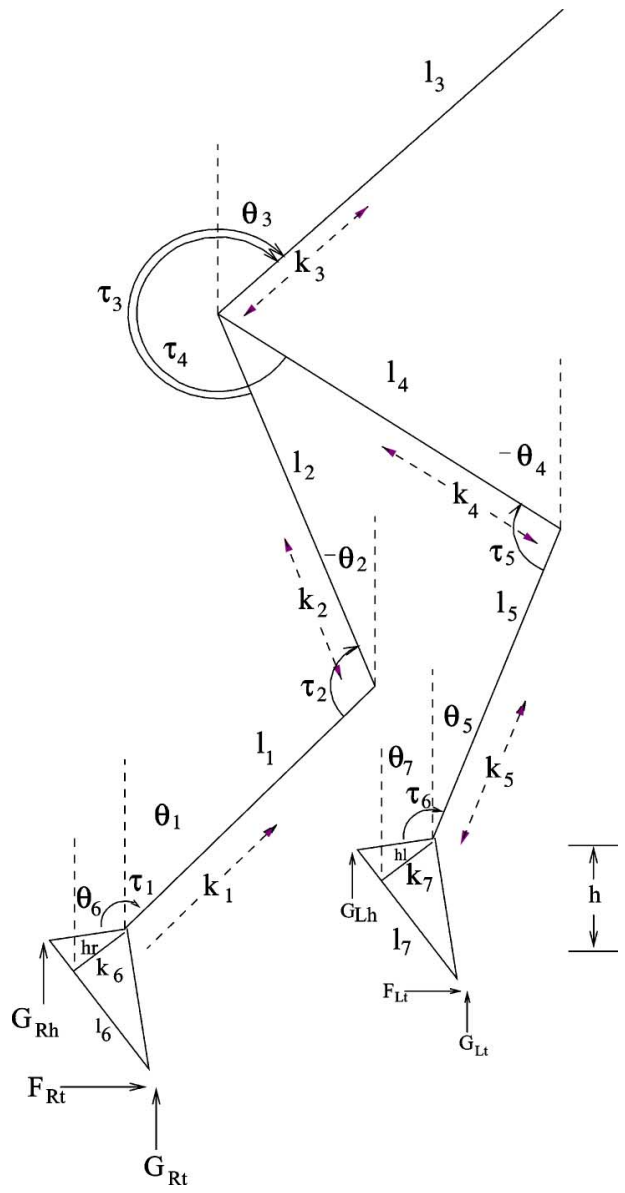

FIGURE 2. The seven-link sagittal biped.

turbance is larger and the biped takes a step forward to keep from falling. These simulations were programmed in $\mathrm{C}++$.

\section{Balance without Stepping}

In this simulation, the biped is subjected to an impulsive force of $39 \mathrm{~N}$ while standing with its feet together. The disturbance force is applied horizontally at the center of mass of the third link. The effect of this theoretical impulse on the system is represented by the instant jumps in the velocities of all segments. The biped responds by moving up onto its toes, raising its center of mass, as shown in Fig. 3, and returns to equilibrium.

TABLE 1. Physical parameters of the seven-link biped.

\begin{tabular}{lccll}
\hline Variable & $M(\mathrm{Kg})$ & $I(\mathrm{~m})$ & $k(\mathrm{~m})$ & $I\left(\mathrm{~kg} \mathrm{~m}^{2}\right)$ \\
\hline Leg & 4 & 0.53 & 0.3 & 0.065 \\
Thigh & 8 & 0.53 & 0.3 & 0.126 \\
Torso & 55 & 0.72 & 0.35 & 3.4 \\
Thigh & 8 & 0.53 & 0.3 & 0.126 \\
Leg & 4 & 0.53 & 0.3 & 0.065 \\
Foot & 1.0 & 0.26 & 0.123 & 0.0015 \\
Foot & 1.0 & 0.26 & 0.123 & 0.0015 \\
\hline
\end{tabular}




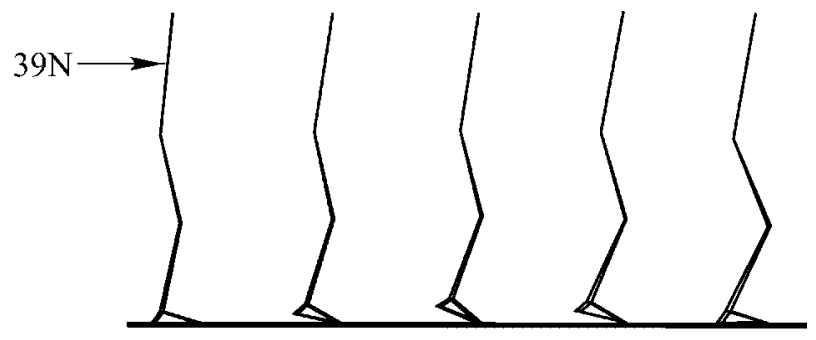

FIGURE 3. The biped rising on tiptoe after an application of an impulsive force to the torso, and returning to its equilibrium.

Table 2 shows the initial positions and velocities of the biped after the impulsive force is applied. Since the center of mass of the biped remains behind the toes, the biped need not step forward to regain its balance.

\section{Stability by Stepping}

When an impulsive force of $45 \mathrm{~N}$ is applied to the biped from behind, the biped moves up on its toes until the center of mass of the biped moves over its toes. At this point, the biped lifts one foot and moves it in front of its center of mass to prevent a fall. The biped first moves up on its toes and then steps forward to keep from falling, as shown in Fig. 4. Table 2 shows the initial positions and velocities of the biped after the impulsive force is applied.

Figure 5 shows the timing schedule for widening the base of support. The dotted line represents the displacement of the horizontal component of the center of mass from the left toe. As it leaves the support base, the right foot is lifted and moved forward to widen the stance as shown by the solid line which represents the right toe.

The trajectories for the angles and angular velocities in this stepping maneuver are, respectively, shown in Figs. 6 and 7.

The magnitude of the disturbance, and its point of application on the body, determine whether a step is necessary. A brief account of this issue is summarized in Table 3 .

TABLE 2. Initial positions and velocities of the biped after a 39-N and a 45-N impulsive push.

\begin{tabular}{|c|c|c|c|c|}
\hline & \multicolumn{2}{|c|}{$39 \mathrm{~N}$} & \multicolumn{2}{|c|}{$45 \mathrm{~N}$} \\
\hline & $\begin{array}{c}\text { Initial } \\
\text { position }\end{array}$ & $\begin{array}{c}\text { Initial } \\
\text { velocity }\end{array}$ & $\begin{array}{c}\text { Initial } \\
\text { position }\end{array}$ & $\begin{array}{c}\text { Initial } \\
\text { velocity }\end{array}$ \\
\hline$\theta_{1}$ & 0.2200 & -0.2026 & 0.2200 & -0.2337 \\
\hline$\theta_{2}$ & -0.2162 & 1.3042 & -0.2162 & 1.5048 \\
\hline$\theta_{3}$ & 0.1000 & 0.3437 & 0.1000 & 0.3966 \\
\hline$\theta_{4}$ & -0.2348 & 1.2926 & -0.2348 & 1.4914 \\
\hline$\theta_{5}$ & 0.2000 & -0.1807 & 0.2000 & -0.2085 \\
\hline$\theta_{6}$ & 0 & -0.5530 & 0 & -0.6381 \\
\hline$\theta_{7}$ & 0 & -0.5823 & 0 & -0.6718 \\
\hline$x_{3}$ & -0.1931 & 0.6513 & -0.1931 & 0.7515 \\
\hline$y_{3}$ & 1.4531 & 0.0325 & 1.4531 & 0.0375 \\
\hline
\end{tabular}



FIGURE 4. The biped rising on tiptoe and taking a step forward after the application of an impulsive force to the torso.

\section{DISCUSSION}

\section{Applications}

The model presented here allows computation of a large number of internal states during specific maneuvers. It can be tuned to a healthy individual by comparing relatively easily measurable physical attributes such as ground reaction forces, other forces of contact with the environment, and possibly EMGs and muscular forces.

The model can be tuned to suffer specified trauma. The clinically known consequences of trauma, such as increases in the gain of short feedback loops of the spindle, or increases in the forward afferent gain of alpha motor neurons can be implemented in the system and simulated. Documented clinical observations on a variety of trauma-related effects can be studied. ${ }^{33}$ In stroke patients, a variety of

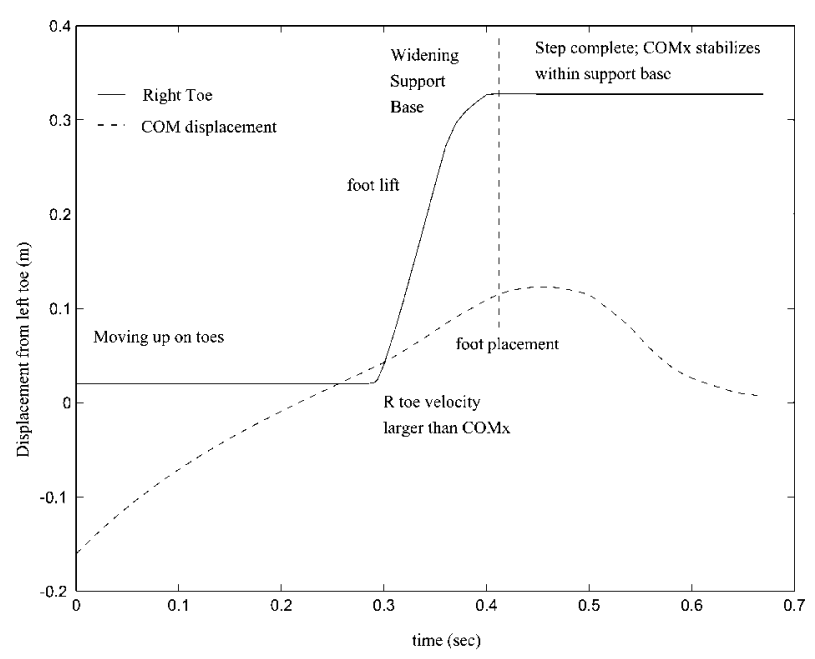

FIGURE 5. The timing schedule for widening the base of support. The dotted line represents the displacement of the horizontal component of the center of mass relative to the left toe. As it leaves the support base, the right foot is lifted and moved forward to widen the stance as shown by the solid line which represents the right toe. In time interval $1,0<t<0.3 \mathrm{~s}$, the biped moves up on its toes, the toes are stationary, and the COM's horizontal component moves beyond the toes. At about $t=0.3$ the right foot lifts. In interval $0.3<t<0.42 \mathrm{~s}$, stepping is initiated. The right foot accelerates and moves faster than the horizontal component of the velocity of the COM. At about $t=$ $0.42 \mathrm{~s}$., the right foot touches the ground. In interval $0.42<t$ $<0.7 \mathrm{~s}$, the step is complete. The center of mass decelerates toward zero velocity. Additional adjustment, not shown, may be needed to get back to the initial equilibrium state. 


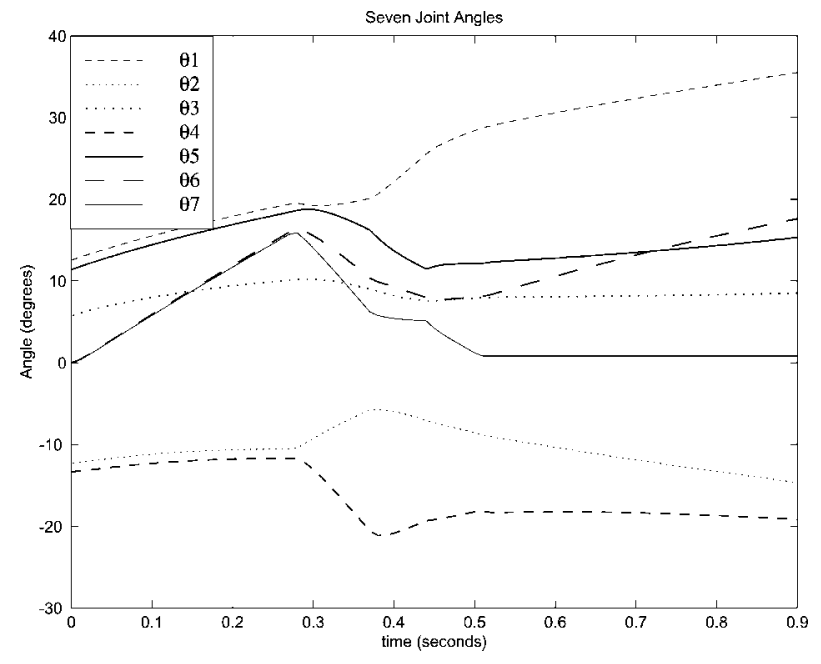

FIGURE 6. The joint angles of the biped as functions of time after it is given a $45 \mathrm{~N}$ push from behind, and it must step forward to keep its balance. The angle of the right foot, i.e., the moving foot, $\left(\theta_{1}\right)$ increases from about $10^{\circ}$ to about $35^{\circ}$, while the angle of the left foot $\left(\theta_{1}\right)$ remains relatively stationary. Similarly, the angles of the right thigh $\left(\theta_{2}\right)$ and the left thigh $\left(\theta_{4}\right)$ remain relatively the same before and after the step. This is not quite natural for humans in a similar situation, but the control strategy works in the sense that the biped, after the step is taken, comes to a stable equilibrium state. The angle of the body $\left(\theta_{5}\right)$, as shown, does not appreciably change during the transition.

deficiencies such as mass synergy patterns, prolonged or premature activity that is unrelated to stretch, spasticity, or stretch dependent overactivity, cocontractions, and, finally, impaired motion dure to increased EMG activity can be simulated, because all the parameters of the model are computable and transparent.



FIGURE 7. The joint angular velocities of the biped after it is given a $45 \mathrm{~N}$ push from behind, and it must step forward to keep its balance. The angular velocities are somewhat jerky and discontinuous, and not probably as smooth as in a similar human step.
TABLE 3. Largest impulsive forces allowable before the biped will step to regain its balance.

\begin{tabular}{lc}
\hline Location of force & Force $(N)$ \\
\hline Head & 30 \\
COM $^{a}$ of torso & 39 \\
Hips & 49 \\
\hline${ }^{a}$ COM: center of mass. &
\end{tabular}

It is possible to introduce hyper- or hypoactivity of individual muscles or groups of muscles, and study the effects of shortening, lengthening, elimination, or atrophy of tendons, etc. This second application may be relevant in the study of Parkinson effects.

The model could also be used to monitor recovery in rehabilitation.

\section{CONCLUSIONS}

Internal models and states were discussed that could be used in voluntary stepping. A sagittal model was presented with certain quantitative results that resemble the hip-ankle foot-in-place and stepping strategies in humans. The control strategy is simple and is the same regardless of the initial velocity of the disturbance. When the disturbance is large enough to warrant a step, the controller initiates one. This strategy can become ineffective if the forward initial velocity of the biped is large enough such that the time used in slowing the velocity by moving up on its toes would be better spent by stepping right away.

The controller needs the internal states of the location and velocity of the center of mass in order to make decisions about stepping and desired foot velocities. The internal states provide such information to the controller about the apparent stability of the biped. The center of pressure can also provide information about the apparent stability of the biped. Because the center of pressure involves both feet, the location of the center of pressure may have to be estimated as another internal state. A more complete foot model with distributed forces could provide better information. The use of muscles, neural transmission latencies, and neural processing elements should make such quantitative models more physiologically accurate, and more medically and diagnostically useful.

\section{REFERENCES}

${ }^{1}$ Anastasio, T. "Vestibuo-ocular reflex." In: The Handbook of Brain Theory and Neural Networks, edited by M. Arbib. Cambridge, MA: The M.I.T. Press, 1995, pp. 1003-1005.

${ }^{2}$ Arbib, M. A., ed. The Handbook of Brain Theory and Neural Networks. Cambridge, MA: The MIT Press, 1995.

${ }^{3}$ Barin, K., and J. Durrant, "Applied physiology of the vestibular system.” In: The Ear: Comprehensive Otology, edited by R. Canalis and P. Lambert. Philadelphia: Lippincott Williams and Wilkins, 2000, pp. 113-140. 
${ }^{4}$ Bay, J. Fundamentals of Linear State Space Systems. Bostan: WCB McGraw-Hill, 1999.

${ }^{5}$ Brooks, V. The Neural Basis of Motor Control. Newyork: Oxford University Press, 1986.

${ }^{6}$ Cannon, S., D. Robinson, and S. Shamma. "A proposed neural network for the integrator of the oculomotor system," Biol. Cybernet. 49:127-136, 1983.

${ }^{7}$ Cooper, L. A., and R. N. Shepard, "Turning something over in the mind." Sci. Am. 251(6):106-114, 1984.

${ }^{8}$ Dietz, V., M. Trippel, and G. Horstmann, "Significance of prioceptive and vestibulo-spinal reflexes in the control of stance and gait.” In: Adaptability of Human Gait, Implications for the Control of Locomotion, edited by A. E. Patla. Amsterdam: North Holland, 1991, pp. 37-52.

${ }^{9}$ Hain, T., T. Ramaswamy, and M. Hillman, "Anatomy and physiology of the normal vestibular system.” In: Vestibular Rehabilitation, edited by S. Herdman. Philadelphia: F. A. Davis Company, 2000, pp. 3-24.

${ }^{10}$ Hemami, H. "Modeling, control, and simulation of human movement," CRC Crit. Rev. Biomed. Eng. 13(1):1-34, 1985.

${ }^{11}$ Hemami, H. "Some physical attributes of postural adjustment." In: Bioinstrumentation: Research, Developments, and Appliacations, edited by D. Wise. Butterworth Publishers, 1990, ch. 34, pp. 1009-1052.

${ }^{12}$ Hemami, H., and A. Katbab, "Constrained inverted pendulum for evaluating upright stability." J. Dyn. Syst. Measure. Control, pp. 343-349, 1982.

${ }^{13}$ Horak, F. B., and L. M. Nashner. "Central programming of postural movements: Adaptation to altered support surface configurations." J. Neurophisiol. 55:1369-1381, 1986.

${ }^{14}$ Horak, F., and C. Shupert, "Role of the vestibular system in postural control." In: Vestibular Rehabilitation, 2nd ed., edited by S. Herdman. Philadelphia: 1994, F. A. Davis Co. pp. 25-51.

${ }^{15}$ Horstmann, G., and V. Dietz, "The basic posture control mechanism: The stabilization of the center of gravity.” Electroencephal Clin Neurophysiol. 76:165-176, 1990.

${ }^{16}$ Jalics, L., H. Hemami, and B. Clymer. "A control strategy for terrain adaptive bipedal locomotion." Autonom. Robots 4:243257, 1997.

${ }^{17}$ Jalics, L., H. Hemami, B. Clymer, and A. Groff, "Rocking, tapping, and stepping, a prelude to dance." Autonom. Robots 4:227242, 1997.

${ }^{18}$ Jian, Y., D. Winter, M. Ishac, and L. Gilhrist. "Trajectory of the body $\operatorname{cog}$ and cop during initiation and termination of gait." Gait Posture 1:9-22, 1993.

${ }^{19}$ Kallel, H. Stability and Control of Constrained ThreeDimensional Robotic Systems with Application to Bipedal Postural Movements. Ph.D. thesis, The Ohio State University, 1991.
${ }^{20}$ Kawato, M. "Cerebellum and motor control." In: The Handbook of Brain Theory and Neural Networks, edited by M. Arbib. Cambridge, MA: The M.I.T. Press, 1995, pp. 172-178.

${ }^{21} \mathrm{Kim}$, J., and H. Hemami, "Coordinated three-dimensional motion of the head and torso by dynamic neural networks." IEEE Trans. Syst. Man Cybernet. B (5):653-666, 1998.

${ }^{22}$ Kuo, A. D., and F. E. Zajac. "Human standing posture: Multijoint movement strategies based on biomechanical constraints." Prog. Brain Res. 97:349-358, 1993.

${ }^{23}$ Latash, M. L. Control of Human Movements. Chicago: Human Kinetics Publishers, 1993.

${ }^{24}$ Luchies, C., N. Alexander, A. Schult, and J. Ashton-Miller. "Stepping responses of young and old adults to postural disturbances: Kinematics." J. Am. Geriatr. Soc. 42:506-512, 1994.

${ }^{25}$ Maki, B. E., W. E. McIlroy, and S. D. Perry. "Influence of Lateral destabilization on compensatory stepping responses." $J$. Biomech. 29(3):343-351, 1996.

${ }^{26}$ Nashner, L., and G. McCollum. "The organization of human postural movements: A formal basis and experimental synthesis." Behv. Brain Sci. 8:132-175, 1985.

${ }^{27}$ Nicholls, J., A. Martin, and B. Wallace, From Neuron to Brain, 3rd ed. Sinauer Associates, Inc., Sunderland, MA, 1992.

${ }^{28}$ Ozbay, H. Introduction to Feedback Control Theory. Boca Raton: CRC Press, 1999.

${ }^{29}$ Pai, Y.-C., and J. Patton. "Center of mass velocty-position predictions of balance control." J. Biomech. 30(4):347-354, 1997.

${ }^{30}$ Peterson, B. "Head movement: Multidimensional modeling." In: The Handbook of Brain Theory and Neural Networks, edited by M. Arbib. Cambridge, MA: The MIT Press, 1995, pp. 450-454.

${ }^{31}$ Rogers, M., T. C. Hain, T. A. Hanke, and I. Janssen, "Stimulus parameters and inertial load: Effects on the incidence of protective stepping responses in healthy human subjects." Arch. Phys. Med. Rehabil. 77:363-368, 1996.

${ }^{32}$ Shupert, F., C. L. Horak, and F. Black. "Hip sway associated with vestibulopathy." J. Vestib. Res. 4(3):231-244, 1994.

${ }^{33}$ Shumway-Cook, A., and M. H. Woollacott, Motor control, Theory and Practical Applications. Philadelphia: Lippincott Williams and Wilkins, 2001.

${ }^{34}$ Smith, O. Feedback Control Systems. New York: McGraw-Hill, 1958.

${ }^{35}$ Thelen, D. G., L. A. Wojcik, A. B. Schultz, J. A. Ashton-Miller, and N. B. Alexander. "Age difference in using a rapid step to regain balance during a forward fall." J. Gerontol. Med. Sci. 52A(1):M8-M13, 1997.

${ }^{36}$ Wilson, V. J., and W. Peterson, "The role of the vestibular system in posture and movement." In: Medical Physiology, edited by V. Mountcastle. St. Louis, MD: The C. V. Mosby Co. 1980, ch. 30, pp. 813-836. 